\title{
ELECTROMAGNETIC-THERMAL ANALYSIS FOR HUMAN EXPOSURE TO HIGH FREQUENCY (HF) RADIATION
}

\author{
DRAGAN POLJAK ${ }^{1}$, MARIO CVETKOVIC $^{1}$, HRVOJE DODIG $^{2} \&$ ANDRES PERATTA $^{3}$ \\ ${ }^{1}$ University of Split, Croatia. \\ ${ }^{2}$ Marine Electronics Center, Split, Croatia. \\ ${ }^{3}$ Wessex Institute of Technology, Southmapton, United Kingdom
}

\begin{abstract}
The paper aims to review some electromagnetic-thermal dosimetry methods for the assessment of human exposure to high frequency (HF) electromagnetic fields. The analysis approaches are based on certain integral/differential equation formulations and related numerical solution procedures for the calculation of specific absorption rate (SAR) and related temperature increase in a tissue. Illustrative computational examples for the human eye and the human brain exposed to HF electromagnetic fields are given in the paper. Also, some numerical results for the transcranial magnetic stimulation (TMS) are presented as an example of biomedical application of electromagnetic fields. The obtained numerical results for SAR are compared against exposure limits proposed by ICNIRP (International Commission on Non Ionizing Radiation Protection).

Keywords: high frequency radiation, human brain, human exposure, human eye, specific absorption rate, temperature increase.
\end{abstract}

\section{INTRODUCTION}

Recent rapid growth of wireless and mobile communication systems has increased the public concern regarding possible radiation hazard. As the dominant and well-established biological effect of high frequency (HF) fields is heating of the tissue the HF exposure assessment is based on the calculation of SAR distribution and related temperature increase in the tissue. Of particular interest is the exposure of the eye and brain, respectively.

There are many papers on HF and thermal dosimetry methods, e.g. [1-5]. As measurement of internal fields and related temperature rise is not possible the theoretical models for the exposure assessment are necessary to simulate various exposure scenarios, and thereby establish safety guidelines and exposure limits for humans [6, 7]. Computational models can be classified as either realistic models of the body (or particular organs) mostly based on Magnetic Resonance Imaging (MRI), e.g. [4], or simplified models, computationally much less demanding but failing to provide accurate results in most of the exposure scenarios [8].

This paper reviews the use of integral, differential and hybrid approaches in bioelectromagnetics and thermal dosimetry developed by the authors.

Computational examples presented in the paper are related to the eye and brain exposure to HF electromagnetic fields and to the calculation of related temperature rise. The eye exposure is based on the hybrid boundary element/finite element method (BEM/FEM) of solution of the Helmholtz [3]. The brain exposure is based on solving the set of coupled surface integral equations (SIEs) via the Method of Moments (MoM). The obtained maximum values of SAR are compared to the exposure limits proposed by ICNIRP [6]. The thermal dosimetry formulation for the eye and brain, respectively, is based on the FEM solution of the stationary Pennes bio-heat equation and related FEM solution $[9,10]$. 


\section{THEORETICAL BACKGROUND FOR ELECTROMAGNETIC-THERMAL DOSIMETRY}

The principal dosimetric quantity for quantifying the influence of HF fields is the specific absorption rate (SAR).

High complexity of the problem was the main reason that the early stage researchers investigated simple models such as plane slab, cylinders, homogeneous and layered spheres and prolate spheroids [8]. On the other hand, the most of the recent anatomically based computational models comprising of cubical cells are dominantly related to the application of the Finite Difference Time Domain (FDTD) methods, e.g. [11]. Nevertheless, the FEM and BEM have been also used, but to a somewhat lesser extent, e.g. [3].

$S A R$ is defined in terms of power $P$ dissipated in the unit body mass $m$ :

$$
S A R=\frac{d P}{d m}=\frac{d}{d m} \frac{d W}{d t}=C \frac{d T}{d t}
$$

where $W$ represents the energy absorbed by the unit body mass, $C$ is the specific heat capacity of tissue, $T$ is the temperature and $t$ denotes time.

$S A R$ is proportional to the square of the internal electric field, as well:

$$
S A R=\frac{d P}{d m}=\frac{d P}{\rho d V}=\frac{\sigma}{2 \rho}|E|^{2}=\frac{\sigma}{\rho}\left|E_{r m s}\right|^{2}
$$

where $E$ and $E_{\text {rms }}$ is the peak and root-mean-square value of the electric field, respectively, $\rho$ is the tissue density and $\sigma$ is the tissue conductivity.

$S A R$ depends on the incident field parameters, characteristics of the exposed body, ground and reflector effects, respectively and generally reaches maximal values when the electric field is oriented parallel to the long body axis.

Once the $S A R$ distribution in a tissue is determined the temperature increase can be obtained by solving the steady-state bio-heat eqn. [10]:

$$
\nabla \cdot(k \nabla T)+\rho_{b} c_{b} w\left(T_{a}-T\right)+Q_{m}+\rho \cdot S A R=0
$$

where $k$ stands for the heat conduction, $\rho_{b}$ is the blood mass density, $c_{b}$ is the specific heat capacity of blood, $w$ is the blood perfusion rate, $T_{a}$ is the arterial temperature, $Q_{m}$ is the tissue dependent heat source due to metabolic processes, while term $\rho \cdot S A R$ represents the volumetric heat source due to an external electromagnetic field.

\section{THE EYE EXPOSURE TO HF FIELDS}

The human eye exposed to plane wave is referred to as an electromagnetic scattering from lossy dielectric object. The eye model is based on the Stratton-Chu formulation and Helmholtz equation for external and internal part of the problem, respectively. The hybrid BEM/ FEM approach with edge elements is applied to treat the eye problem.

\subsection{Electromagnetic dosimetry}

Plane wave incident on the corneal part of the eye can be treated as an unbounded scattering problem, as shown in Fig. 1. The eye is discretized to 36,027 tetrahedral elements. 


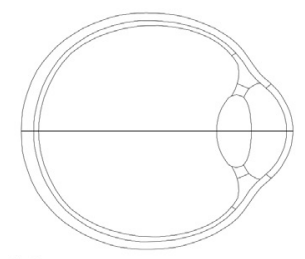

(a)

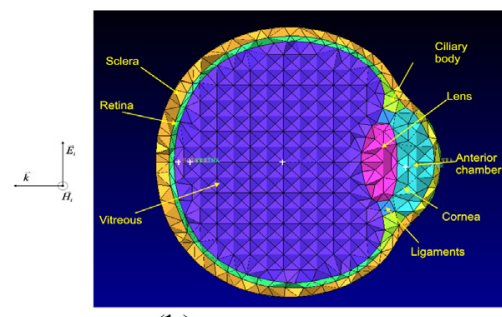

(b)

Figure 1: (a) Model of the eye exposed to plane wave (b) Meshing detail.

According to Stratton-Chu formulation, the time-harmonic electric field occurring at the exterior of the scattering problem is governed by the following boundary integral eqn. [3]:

$$
a \vec{E}=\vec{E}_{i}+\oint_{\partial V^{\prime}} \vec{n}^{\prime} \times\left(\nabla^{\prime} \times \vec{E}\right) G d S^{\prime}+\oint_{\partial V^{\prime}}\left[\left(\vec{n}^{\prime} \times \vec{E}\right) \times \nabla^{\prime} G+\left(\vec{n}^{\prime} \cdot \vec{E}\right) G\right] d S^{\prime}
$$

where $\vec{E}_{i}$ is the incident field, $\vec{n}^{\prime}$ is an outer normal to surface $\partial V^{\prime}$ bounding the volume $V$ and $\alpha$ is the solid angle subtended at an observation point, while $G$ represents the fundamental solution of the corresponding Helmholtz equation:

$$
\nabla^{2} G+k^{2} G=-\delta\left(\vec{r}-\vec{r}^{\prime}\right)
$$

Furthermore, the interior of the eye containing inhomogeneous regions is represented by the Helmholtz type equation of the form [3]:

$$
\nabla \times\left(\frac{1}{k_{B}} \nabla \times \vec{E}\right)-k_{A} \vec{E}=0
$$

where subscripts A and B denote the exterior and interior region, respectively.

The governing equations of the electromagnetic model of the eye are solved using the hybrid BEM/FEM approach presented in [3].

Applying the weighted residual approach to eqn (6) the following integral representation is obtained [3]:

$$
\int_{V^{\prime}}\left[\nabla \times\left(\frac{1}{k_{B}} \nabla \times \vec{E}\right)-k_{A} \vec{E}\right] \cdot \vec{W}_{j} d V^{\prime}=0
$$

where vector test functions $\vec{W}_{j}$ denotes the set of vector test functions.

Once the electric field $\vec{E}$ is known $S A R$ can be computed from (2) while the related temperature increase is obtained by solving the Pennes eqn (3) via FEM, as outlined in the Appendix.

\subsection{Numerical results}

Figures 2 and 3 show numerical results for the SAR in the eye exposed to plane wave with power density of $10 \mathrm{~W} / \mathrm{m}^{2}$ at different frequencies. The key physical properties of the eye model are available in [9]. 
The maximum values of SAR at different frequencies, averaged over the whole eye, are presented in Table 1.

The results show that as frequency increases SAR distribution becomes more localised, promoting the formation of 'hot spots' between 1 and $4 \mathrm{GHz}$. Also, the whole eye averaged SAR values stay below the ICNIRP exposure limits for localized SAR in the head for general public population $(2 \mathrm{~W} / \mathrm{kg})[6]$.

Figures 4 and 5 show the related temperature increase in the eye due to the induced SAR presented in Figs 2 and 3, respectively.

The obtained maximum temperature increase is $0.1^{\circ} \mathrm{C}$ at $4 \mathrm{GHz}$ while the hot spot region is within the vitreous body as the absorbed energy is focused in vitreous region at $4 \mathrm{GHz}$, and there is also a lack of the blood perfusion within vitreous body.

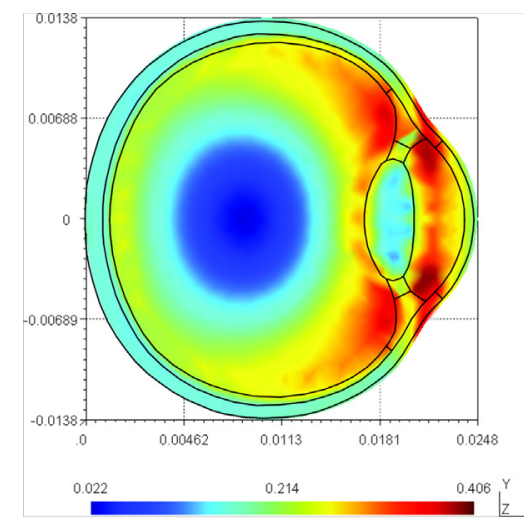

(a)

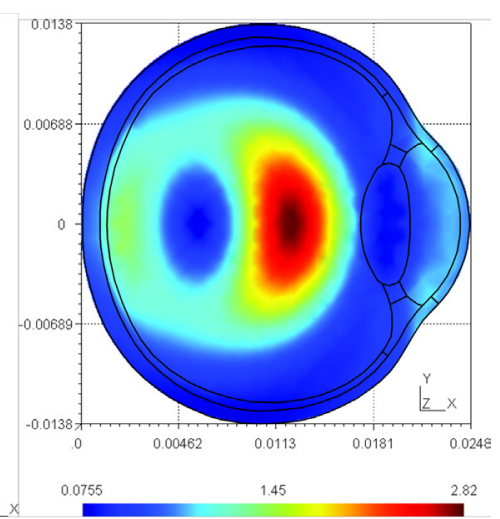

(b)

Figure 2: SAR in the eye exposed to plane wave of power density $10 \mathrm{~W} / \mathrm{m}^{2}$ at frequency (a) $1 \mathrm{GHz}$ (b) $2 \mathrm{GHz}$.

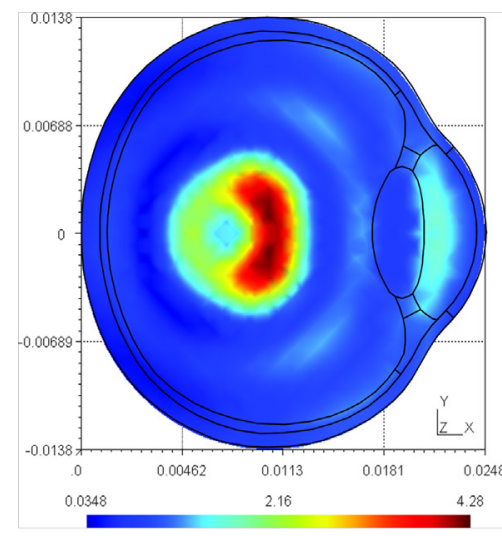

(a)

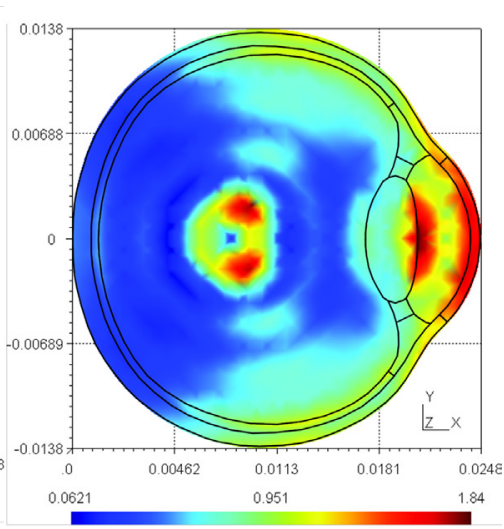

(b)

Figure 3: SAR in the eye exposed to plane wave of power density $10 \mathrm{~W} / \mathrm{m}^{2}$ at frequency (a) $4 \mathrm{GHz}$ (b) $6 \mathrm{GHz}$. 
Table 1: The whole eye averaged SAR at different frequencies.

\begin{tabular}{ll}
\hline Frequency[GHz] & SAR $[\mathrm{W} / \mathrm{kg}]$ \\
\hline 1 & 0.3352 \\
2 & 0.6189 \\
4 & 1.2617 \\
6 & 1.0689 \\
\hline
\end{tabular}

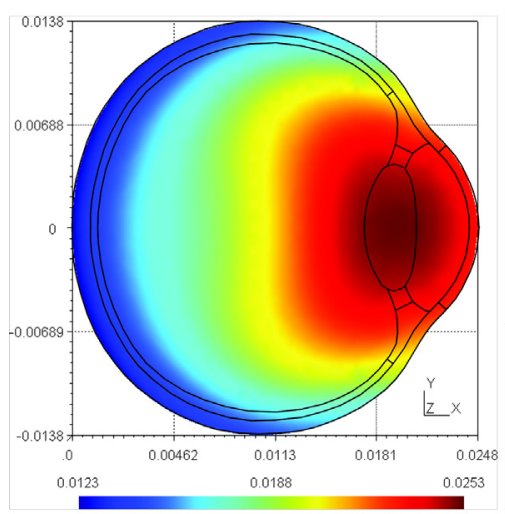

a)

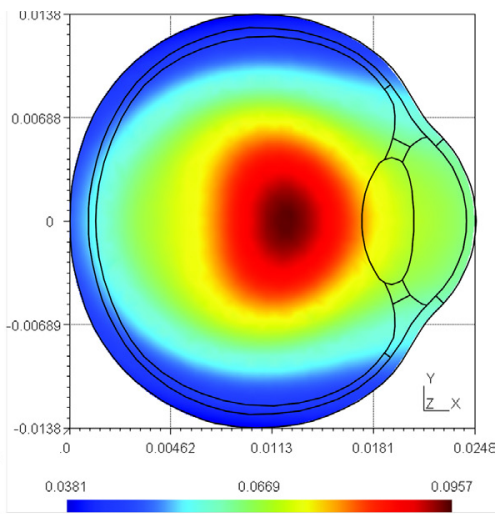

b)

Figure 4: Temperature increase in the eye exposed to plane wave of power density $\mathrm{P}=10 \mathrm{~W} / \mathrm{m}^{2}$ at frequency (a) $1 \mathrm{GHz}$ (b) $2 \mathrm{GHz}$.

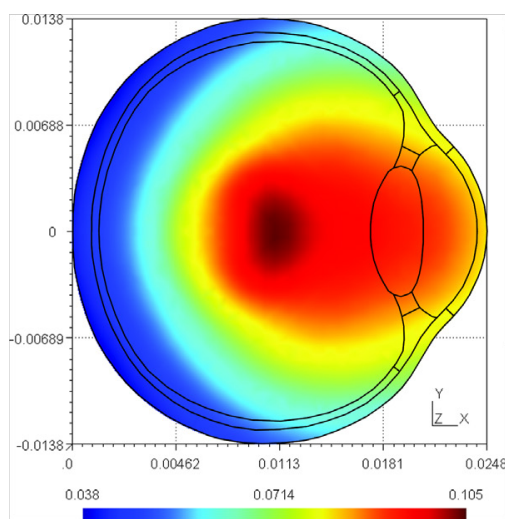

(a)

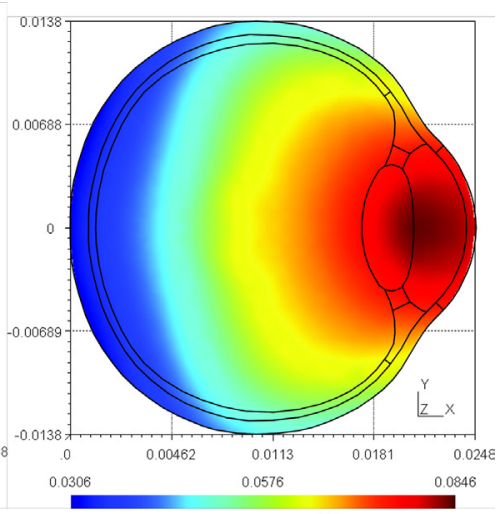

(b)

Figure 5: Temperature increase in the eye exposed to plane wave of power density $\mathrm{P}=10 \mathrm{~W} / \mathrm{m}^{2}$ at frequency (a) $4 \mathrm{GHz}$ (b) $6 \mathrm{GHz}$. 
It is worth noting that the SAR results presented in this paper are in a good agreement with the results reported in [5].

\section{THE BRAIN EXPOSURE TO HF RADIATION}

The lossy dielectric model of the human brain exposed to HF radiation is based on the SIE approach [12]. The formulation itself is usually derived from the equivalence theorem and by using the appropriate interface conditions for the electric and/or magnetic field, as depicted in Fig. 6.

The lossy homogeneous object representing the brain is illuminated by the incident electromagnetic field $\left(\vec{E}^{i n c} ; \vec{H}^{i n c}\right)$.

\subsection{Dosimetry}

Using the equivalence theorem, two problems are formulated, in terms of the equivalent electric and magnetic current densities $\vec{J}$ and $\vec{M}$ existing at the surface $S$, one for the region 1 (exterior to dielectric) and other for the region 2 (inside the dielectric) [12].

The boundary conditions at the surface $S$ are satisfied by introducing equivalent surface currents $\vec{J}_{2}=-\vec{J}_{1}$ and $\vec{M}_{2}=-\vec{M}_{1}$. Using the same procedure for the interior equivalent problem, yields another homogeneous domain introducing the equivalent surface currents $\vec{J}_{1}$ and $\vec{M}_{1}$.

Performing some mathematical manipulations, the following set of integral equations is obtained [12]:

$$
\begin{aligned}
& j \omega \mu_{n} \iint_{S} \vec{J}\left(\vec{r}^{\prime}\right) G_{n}\left(\vec{r}, \vec{r}^{\prime}\right) d S^{\prime}- \\
& -\frac{j}{\omega \varepsilon_{n}} \iint_{S} \nabla_{S}^{\prime} \cdot \vec{J}\left(\vec{r}^{\prime}\right) \nabla G_{n}\left(\vec{r}, \vec{r}^{\prime}\right) d S^{\prime}+ \\
& +\iint_{S} \vec{M}\left(\vec{r}^{\prime}\right) \times \nabla^{\prime} G_{n}\left(\vec{r}, \vec{r}^{\prime}\right) d S^{\prime}= \begin{cases}\vec{E}^{i n c}, & n=1 \\
0, & n=2\end{cases}
\end{aligned}
$$

where the Green's function for the homogeneous medium is given by:

$$
G_{n}\left(\vec{r}, \vec{r}^{\prime}\right)=\frac{e^{-j k_{n} R}}{4 \pi R} ; \quad R=\left|\vec{r}-\vec{r}^{\prime}\right|
$$

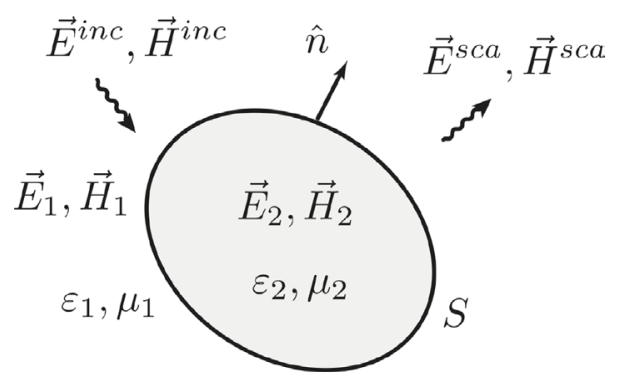

Figure 6: The brain represented by a lossy homogeneous dielectric. 


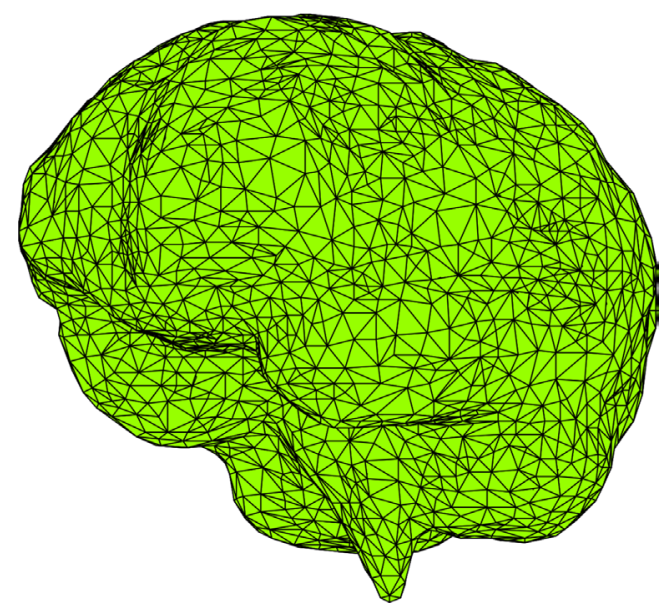

Figure 7: Triangular mesh of the homogeneous brain model.

and $R$ is the distance from the source to observation point, respectively, while $k_{\mathrm{n}}$ is the wave number of a medium $n,(n=1 ; 2)$.

The set of the coupled SIEs (8) is solved via the MoM. Figure 7 shows a view to the triangular brain model mesh.

As a first step, the equivalent electric and magnetic currents $\vec{J}$ and $\vec{M}$ in eqn (8) are expressed in terms of a linear combination of basis functions $\vec{f}_{n}$ and $\vec{g}_{n}$, respectively.

$$
\begin{gathered}
\vec{J}(\vec{r})=\sum_{n=1}^{N} J_{n} \vec{f}_{n}(\vec{r}) \\
\vec{M}(\vec{r})=\sum_{n=1}^{N} M_{n} \vec{g}_{n}(\vec{r})
\end{gathered}
$$

where $J_{\mathrm{n}}$ and $M_{\mathrm{n}}$ are unknown coefficients, while $N$ is the total number of triangular elements.

Applying the weighted residual approach, i.e. multiplying the integral eqn. (8) by the set of a test functions $\vec{f}_{m}$ and integrating over the surface $S$, performing some mathematical manipulations, it follows:

$$
\begin{aligned}
& j \omega \mu_{i} \sum_{n=1}^{N} J_{n} \iint_{S} \vec{f}_{m}(\vec{r}) \cdot \iint_{S^{\prime}} \vec{f}_{n}\left(\vec{r}^{\prime}\right) G_{i} d S^{\prime} d S+ \\
& +\frac{j}{\omega \varepsilon_{i}} \sum_{n=1}^{N} J_{n} \iint_{S} \nabla_{S} \vec{f}_{m}(\vec{r}) \iint_{S^{\prime}} \nabla_{S^{\prime}} \vec{f}_{n}\left(\vec{r}^{\prime}\right) G_{i} d S^{\prime} d S+ \\
& \pm \sum_{n=1}^{N} M_{n} \iint_{S} \vec{f}_{m}(\vec{r}) \cdot\left[\vec{n} \times \vec{g}_{n}\left(\vec{r}^{\prime}\right)\right] d S+ \\
& +\sum_{n=1}^{N} M_{n} \iint_{S} \vec{f}_{m}(\vec{r}) \cdot \iint_{S^{\prime}} \vec{g}_{n}\left(\vec{r}^{\prime}\right) \times \nabla^{\prime} G_{i} d S^{\prime} d S= \\
& = \begin{cases}\iint_{S} \vec{f}_{m}(\vec{r}) \cdot \vec{E}^{i n c} d s \quad, i=1 \\
0 \quad, i=2\end{cases}
\end{aligned}
$$


where subscript $i$ denotes the index of the medium. The details of the numerical solution procedure could be found elsewhere, e.g. in [12].

\subsection{Numerical results}

Computational examples are related to the brain exposed to plane wave at $f=900 \mathrm{MHz}$ and $1,800 \mathrm{MHz}$, respectively. The brain surface is disretised using 696 triangular elements and 1,044 edge-elements while the corresponding brain parameters can be found in [12].

The power density of the incident plane wave is $P=5 \mathrm{~mW} / \mathrm{cm}^{2}$ (oriented perpendicular to the right side of the brain - positive $\mathrm{x}$ coordinate).

Figures 8-11 show the $S A R$ distribution and related temperature increase in the brain at $f=$ $900 \mathrm{MHz}$ and $f=1,800 \mathrm{MHz}$ for the case of horizontal and vertical polarization, respectively.

The obtained peak and average $S A R$ values for both polarization types and for $f=900 \mathrm{MHz}$ and $f=1800 \mathrm{MHz}$ are given in Table 2 .
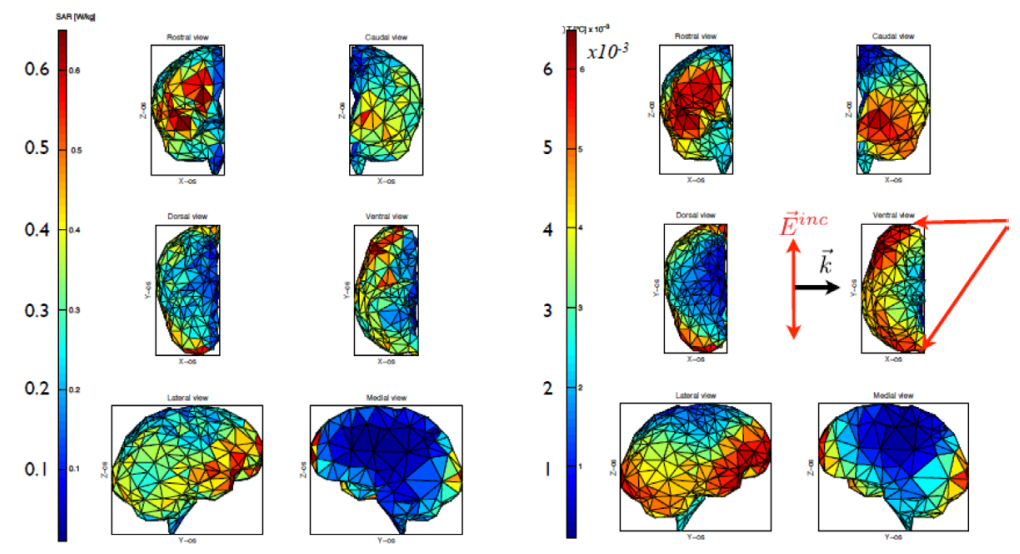

Figure 8: SAR distribution and temperature increase at $f=900 \mathrm{MHz}$ - horizontal polarization.
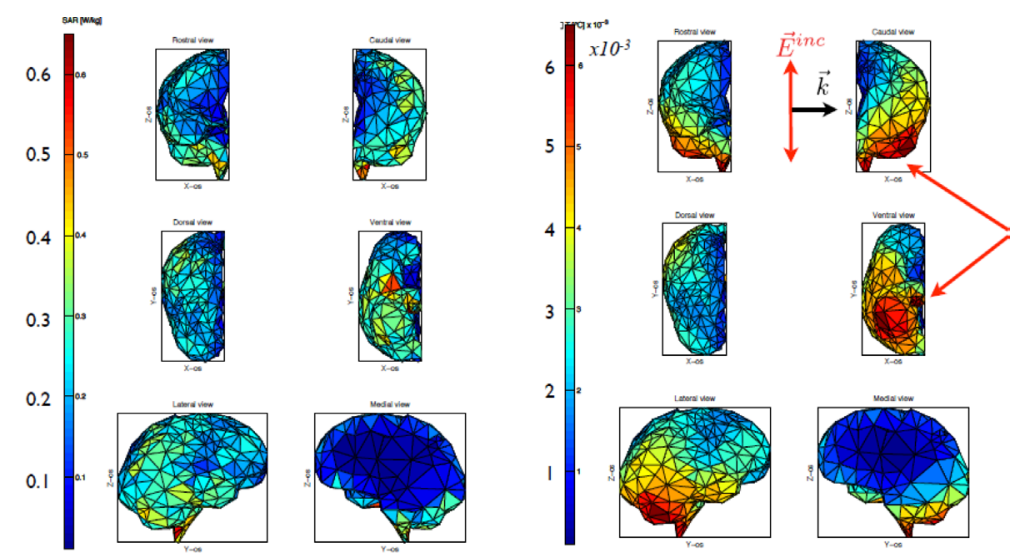

Figure 9: $S A R$ distribution and temperature increase at $f=900 \mathrm{MHz}$ - vertical polarization. 

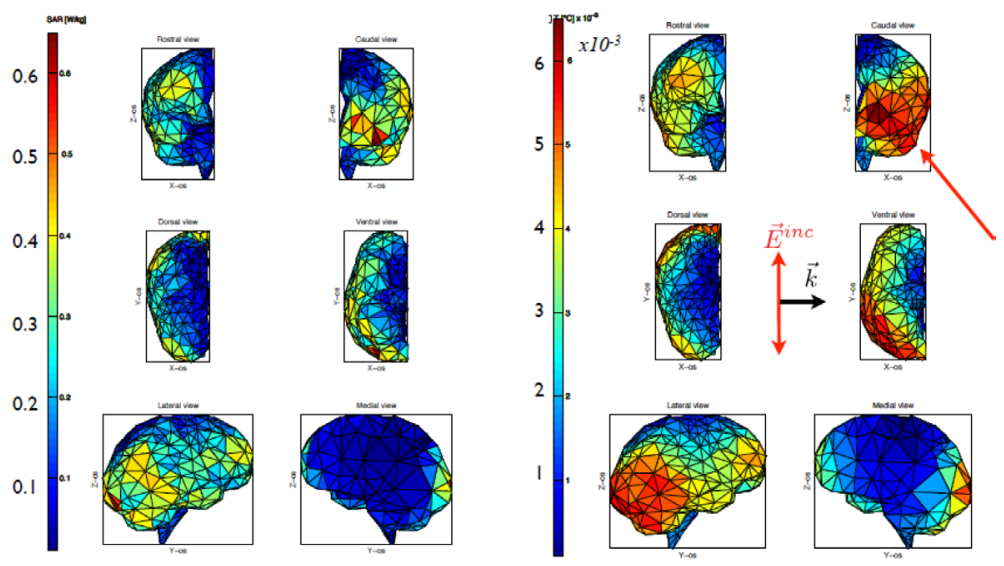

Figure 10: $S A R$ distribution and temperature increase at $f=1,800 \mathrm{MHz}$ - horizontal polarization.
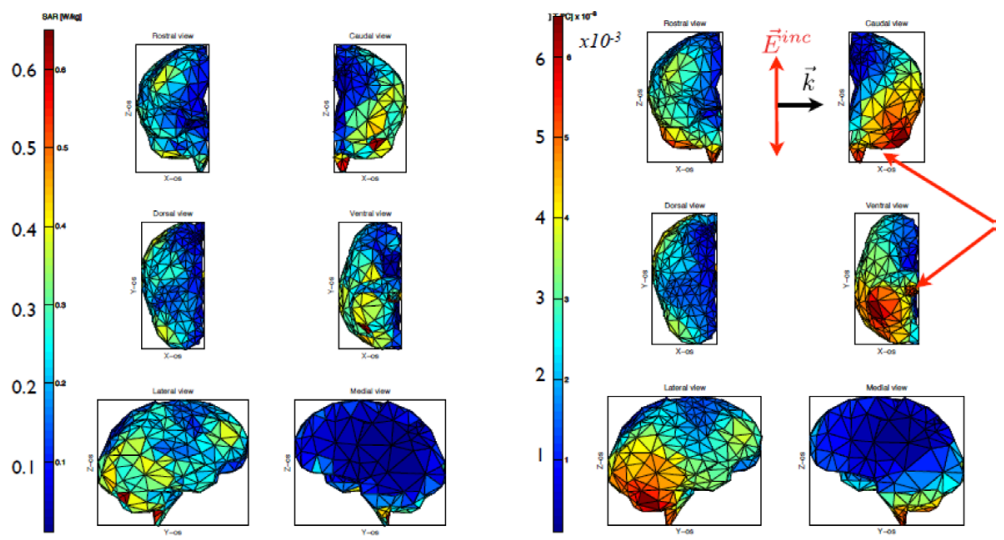

Figure 11: $S A R$ distribution and temperature increase at $f=1,800 \mathrm{MHz}$ - vertical polarization.

Table 2: The peak and average $S A R$ values for different exposure scenarios.

\begin{tabular}{lcccc}
\hline & \multicolumn{2}{c}{$\mathrm{H}$} & \multicolumn{2}{c}{$\mathrm{V}$} \\
\cline { 2 - 5 } & $900 \mathrm{MHz}$ & $1,800 \mathrm{MHz}$ & $900 \mathrm{MHz}$ & $1,800 \mathrm{MHz}$ \\
\hline $\mathrm{SAR}_{\text {max }}[\mathrm{W} / \mathrm{kg}]$ & 0,856486 & 4,390451 & 0,866016 & 2,678407 \\
$\mathrm{SAR}_{a v g}[\mathrm{~W} / \mathrm{kg}]$ & 0,174457 & 0,411736 & 0,158206 & 0,348032 \\
\hline
\end{tabular}

The obtained peak SAR values in the brain do not exceed the ICNIRP limits [6] for localized SAR in the head averaged per $10 \mathrm{~g}$ of tissue (10 W/kg for the occupational exposure).

However, the exposure limit for the general public exposure limit $(2 \mathrm{~W} / \mathrm{kg}$ localized in the head and trunk) has been exceeded at $f=1,800 \mathrm{MHz}$ for both polarizations. 
The whole brain averaged $S A R$ at $f=900 \mathrm{MHz}$ is $0.17 \mathrm{~W} / \mathrm{kg}$ which stay below the ICNIRP exposure limits for localized SAR in the head of $2 \mathrm{~W} / \mathrm{kg}$ averaged per $10 \mathrm{~g}$ of tissue [6]. The maximum temperature increase in the brain is found to be less than $0.01^{\circ} \mathrm{C}$.

Finally, the last set of numerical results are related to transcranial magnetic stimulation (TMS), a non-invasive and painless technique for stimulation or inhibition of certain regions in the human brain [13].

The computational examples for TMS are based on three generic coils used in TMS, namely, the standard circular coil, the figure-of-eight coil (8-coil), and the butterfly coil (8-coil with wings inclined at 10 degrees). The circular coil is discretized into 80 linear segments, the same as the wings of other two coils. The parameters of the three coils are given in Table 3.

In all cases, the coils were placed over the primary motor cortex area, with a distance of $1 \mathrm{~cm}$ between surface of the brain and the geometric center of the coil.

Figure 12 shows the electric field induced along the surface of the brain due to the electromagnetic field generated by the standard circular coil, the figure-of-eight coil (8-coil), and the butterfly coil. Note that all coils are located $1 \mathrm{~cm}$ over the primary motor cortex.

The Maximum values of the induced electric field are given in Table 4.

Table 3: The coil parameters.

\begin{tabular}{lccc}
\hline Frequency & $2.44 \mathrm{kHz}$ & $2.44 \mathrm{kHz}$ & $2.44 \mathrm{kHz}$ \\
Radius of turn & $4.5 \mathrm{~cm}$ & $3.5 \mathrm{~cm}$ & $3.5 \mathrm{~cm}$ \\
No. of turns & 14 & 15 & 15 \\
Coil current & $2,843 \mathrm{~A}$ & $2,843 \mathrm{~A}$ & $2,843 \mathrm{~A}$ \\
\hline
\end{tabular}
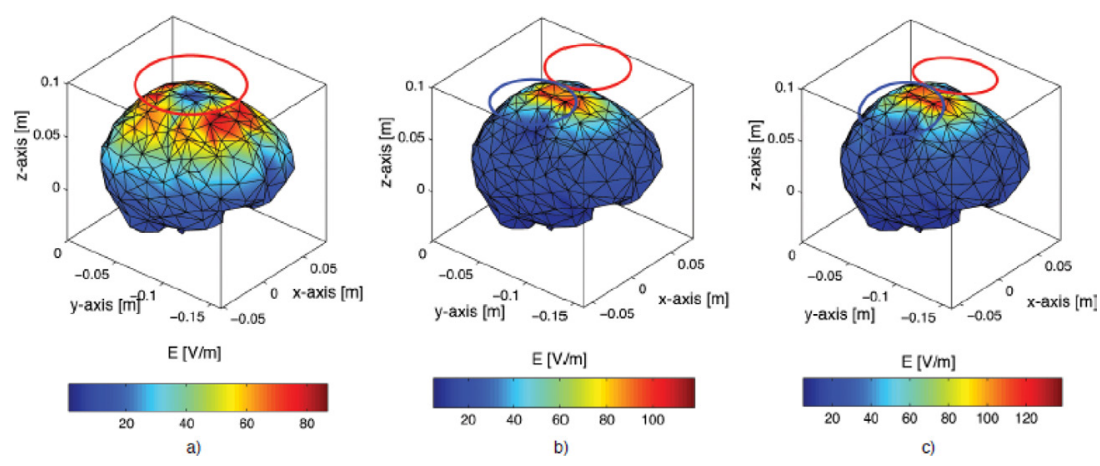

Figure 12: Induced fields along the brain surface due to the field generated by different type of a coil.

Table 4: Maximum values of the induced electric field in the brain.

\begin{tabular}{llll}
\hline & Circular & 8-coil & Butterfly \\
$\mathrm{E}_{\text {max }}[\mathrm{V} / \mathrm{m}]$ & 86.8302 & 118.2815 & 138.4188 \\
\hline
\end{tabular}


More details on TMS modeling via SIEs approach and related MoM solution can be found in [13].

\section{CONCLUSION}

The paper reviews the use of integral, differential and hybrid approaches in HF electromagnetic and thermal dosimetry, respectively, applied to the assessment of the exposure of the human eye and the human brain to HF radiation. The exposure scenarios are analyzed by solving the governing equations arising from the electromagnetic dosimetry via the hybrid BEM/FEM method (Helmholtz equation) and MoM approach (set of SIEs). The thermal dosimetry model is based on the stationary Pennes equation which is treated by FEM. Illustrative computational examples are related to the assessment of SAR in the eye and brain due to the plane wave exposure and to the induced field in the brain due to the TMS treatment. The obtained numerical results are compared against exposure limits proposed by ICNIRP.

\section{REFERENCES}

[1] Hand, J.W., Modeling the interaction of electromagnetic fields (10 MHz-10 GHz) with the human body: methods and applications. Physics in Medicine and Biology, 53(16), pp. 243-286, 2008. http://dx.doi.org/10.1088/0031-9155/53/16/R01

[2] Poljak, D., Electromagnetic fields: environmental exposure. In Encyclopedia of Environmental Health, ed. J.O. Nriagu, Elsevier: Burlington, 2, pp. 259-268, 2011. http://dx.doi.org/10.1016/B978-0-444-52272-6.00422-0

[3] Poljak, D., Cavka, D., Dodig, H., Peratta, C. \& Peratta, A., On the use of boundary element analysis in bioelectromagnetics. Engineering Analysis with Boundary Elements, 49, pp. 2-14, 2014. http://dx.doi.org/10.1016/j.enganabound.2014.02.008

[4] Singh, K.D., Longan, N.S. \& Gilmartin, B., Three dimensional modeling of the human eye based on magnetic resonance imaging. Investigative Opthamology and Visual Science, 47, pp. 2272-2279, 2006. http://dx.doi.org/10.1167/iovs.05-0856

[5] Hirata, A., Temperature increase in human eyes due to near-field and far-field exposures at $900 \mathrm{MHz}, 1.5 \mathrm{GHz}$, and $1.9 \mathrm{GHz}$. IEEE Transactions on Electromagnetic Compatibility, 47(1), pp. 68-76, 2005. http://dx.doi.org/10.1109/TEMC.2004.842113

[6] International Commission on Non-Ionizing Radiation Protection, Guidelines for limiting exposure to time-varying electric, magnetic and electromagnetic fields (up to 300 GHZ). Health Physics, 74(4), pp. 494-522, 1998.

[7] International Commission on Non-Ionizing Radiation Protection, Guidelines for limiting exposure to time-varying electric and magnetic fields (1 HZ - $100 \mathrm{kHZ})$. Health Physics, 99(6), pp. 818-836, 2010.

[8] Poljak, D., Human Exposure to Electromagnetic Fields, WIT Press: Southampton-Boston, 2003.

[9] Dodig, H., Poljak, D. \& Peratta, A., Hybrid BEM/FEM edge element computation of the thermal rise in the 3D model of thehuman eye induced by high frequency em waves. International Conference on Software, Telecommunications and Computer Networks, Split, 2012. 
[10] Poljak, D., Dodig, H., Cavka, D. \& Peratta, A., Some numerical methods of thermal dosimetry for applications in bioelectromagnetics. Proceeding Heat Transfer, Split, Croatia, pp. 271-280, 2012. http://dx.doi.org/10.2495/ht120231

[11] Fujimoto, M., Hirata, A., Wang, J., Fujiwara, O. \& Shiozawa, T., FDTD-derived correlation of maximum temperature increase and peak SAR in child and adult head models due to dipole antenna. IEEE Transactions on Electromagnetic Compatibility, 48(1), pp. 240-247, 2006.

http://dx.doi.org/10.1109/TEMC.2006.870816

[12] Cvetković, M. \& Poljak, D., An efficient integral equation based dosimetry model of the human brain. Proceeding of the 2014 International Symposium on Electromagnetic Compatibility (EMC Europe 2014), Gothenburg, Sweden, pp. 375-380, 2014. http://dx.doi.org/10.1109/emceurope.2014.6930935

[13] Cvetković, M., Poljak, D. \& Haueisen, J., Analysis of transcranial magnetic stimulation based on the surface integral equation formulation, IEEE Transactions on Biomedical Engineering, 62(6), pp. 1535-1545, 2015.

http://dx.doi.org/10.1109/TBME.2015.2393557

\section{APPENDIX: SOLUTION OF BIO-HEAT TRANSFER EQUATION}

The temperature increase in a tissue exposed to external HF fields is obtained by solving the bio-heat transfer equation:

$$
\nabla \cdot(k \nabla T)+\rho_{b} c_{b} w\left(T_{a}-T\right)+Q_{m}+\rho \cdot S A R=0
$$

via FEM.

The integral formulation of (A.1) convenient for FEM solution is given by [10]:

$$
\begin{aligned}
\int_{V^{\prime}} & {\left[k \nabla f_{j} \cdot \nabla T+\rho_{b} c_{b} w \cdot T \cdot f_{j}\right] d V^{\prime} } \\
& =\int_{V^{\prime}}\left(\rho_{b} c_{b} w \cdot T_{a}+Q_{m}+\rho \cdot S A R\right) \cdot f_{j} d V^{\prime}+\oint_{\partial V^{\prime}} k f_{j} \nabla T \cdot d \vec{S}^{\prime}
\end{aligned}
$$

The appropriate boundary condition at the interface between skin and air is:

$$
q=H\left(T_{s}-T_{a}\right)
$$

where $q$ is the heat flux density:

$$
q=-\lambda \frac{\partial T}{\partial n}
$$

while $H, T_{s}$ and $T_{a}$ denote, respectively, the convection coefficient, the temperature of the skin, and the temperature of the air.

The standard finite element discretization of Helmholtz equation yields the following matrix equation:

$$
[K]\{T\}=\{M\}+\{P\}
$$


where $[\mathrm{K}]$ is the finite element matrix given by:

$$
K_{j i}=\int_{\Omega_{e}} \nabla f_{j}\left(\lambda \nabla f_{i}\right) d \Omega_{e}+\int_{\Omega_{e}} W_{b} C_{p b} f_{j} f_{i} d \Omega_{e}
$$

while $\{M\}$ denotes the flux vector:

$$
M_{j}=\int_{\Gamma_{e}} \lambda \frac{\partial T}{\partial n} f_{j} d \Omega_{e}
$$

and $\{P\}$ stands for the source vector:

$$
P_{j}=\int_{\Omega_{e}}\left(W_{b} C_{p b} T_{a}+Q_{m}+\rho \cdot S A R\right) \cdot f_{j} d \Omega_{e}
$$

More details can be found elsewhere, e.g. in $[9,10]$. 